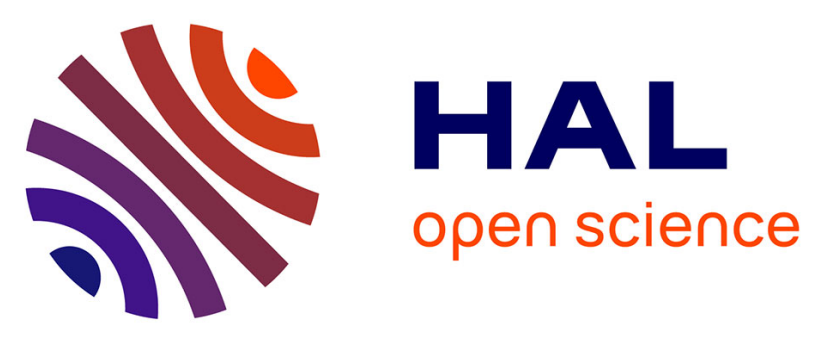

\title{
New insights into the role of Fur proteins: FurB (All2473) from Anabaena protects DNA and increases cell survival under oxidative stress
}

Sara López-Gomollón, Emma Sevilla, M. Teresa Bes, M. Luisa Peleato, María F Fillat

\section{To cite this version:}

Sara López-Gomollón, Emma Sevilla, M. Teresa Bes, M. Luisa Peleato, María F Fillat. New insights into the role of Fur proteins: FurB (All2473) from Anabaena protects DNA and increases cell survival under oxidative stress. Biochemical Journal, 2009, 418 (1), pp.201-207. 10.1042/BJ20081066 . hal00479030

\section{HAL Id: hal-00479030 \\ https://hal.science/hal-00479030}

Submitted on 30 Apr 2010

HAL is a multi-disciplinary open access archive for the deposit and dissemination of scientific research documents, whether they are published or not. The documents may come from teaching and research institutions in France or abroad, or from public or private research centers.
L'archive ouverte pluridisciplinaire HAL, est destinée au dépôt et à la diffusion de documents scientifiques de niveau recherche, publiés ou non, émanant des établissements d'enseignement et de recherche français ou étrangers, des laboratoires publics ou privés. 
New insights into the role of Fur proteins: FurB (All2473) from Anabaena protects DNA and increases cell survival under oxidative stress

\title{
Sara LÓPEZ-GOMOLLÓN, Emma SEVILLA, M. Teresa BES, M. Luisa PELEATO and María F. FILLAT
}

From Department of Biochemistry and Molecular and Cell Biology, and Biocomputation and Complex Systems Physics Institute (BiFi),

University of Zaragoza, Zaragoza, Spain.

\author{
Running head: Role of Fur in oxidative stress response \\ Address correspondence to: María F. Fillat, University of Zaragoza, Pedro Cerbuna, 12, 50009 Zaragoza. Spain. \\ Tel: +34 976761282; Fax: +34 976762123; E-mail: fillat@unizar.es
}

The ferric uptake regulator (Fur) is a prokaryotic transcriptional regulator that controls a large number of genes mainly related to iron metabolism. Frequently, several Fur homologues with different physiological roles are found in the same organism. The genome of the filamentous cyanobacterium Anabaena (Nostoc) sp. PCC 7120 codes for three different fur genes. FurA is an essential protein involved in iron homeostasis that also modulates dinitrogen fixation. FurA interacts with heme, impairing its DNA-binding ability. To explore functional differences between Fur homologues in Anabaena, factors affecting their regulation, as well as some biochemical characteristics have been investigated. While incubation of FurB with heme severely hinders it ability to interact with DNA, binding of heme to FurC could not be detected. Oxidative stress enhances the transcription of the three fur genes, especially of $f u r B$ and $f u r C$. In addition, overexpression of FurA and FurB in $E$. coli increases survival when the cells are challenged with hydrogen peroxide or methyl viologen, a superoxide anion-generating reagent. When present in saturating concentrations, FurB exhibits unspecific DNA-binding activity and protects DNA from cleavage produced by hydroxyl radicals or DNaseI. Based on these results, it can be suggested that whereas at low concentration FurB would act as a member of the Fur family, at saturating concentrations FurB protects DNA, showing a Dps (DNA protection during starvation)-like behaviour.

Keywords: ferric uptake regulator, heme, cyanobacteria, DNA protection, oxidative stress

Abbreviations: Fur, ferric uptake regulator; Dps, DNA protection during starvation; PSI, photosystem I; PerR, peroxide-sensing repressor; bp, base pair(s); DTT, 1,4-dithiothreitol; LB, Luria-Bertani; ORF, open reading frame; ROS, reactive oxygen species; IPTG, isopropyl- $\beta$-Dthiogalactopyranoside; EMSA, electrophoretic mobility shift assays; MV, methyl viologen (paraquat); SDS/PAGE, Sodium dodecyl sulfate polyacrylamide gel electrophoresis.

\section{INTRODUCTION}

Iron participates in numerous metabolic processes and is essential for most living organisms [1]. However, the low solubility of iron in oxygenated waters limits its bioavailability in aquatic environments. This fact, besides the close relationship between iron homeostasis and oxidative stress, make necessary a tight regulation of iron uptake and storage. The ferric uptake regulator (Fur) protein is one of the most important switches for the regulation of iron metabolism in prokaryotes. Under iron-rich conditions, a dimer of Fur binds to specific DNA sequences ("iron-boxes") located in the promoters of iron responsive genes using Fe (II) as co-repressor and avoiding their transcription [2].

Apart from the main group of iron-dependent Fur proteins, the ability of some members of this family to sense other metal ions permits differentiate several subclasses, namely sensors of zinc (Zur) [3], manganese (Mur) [4] or nickel (Nur) [5]. Moreover, some of those Fur-like proteins respond to signals other than metal ions. In fact, using metal-catalyzed protein oxidation some of them regulate DNA-binding activity and thereby respond to the presence of peroxide (PerR) [6] or heme and oxygen (Irr) [7]. The crystal structures of Fur from P. aeruginosa, PerR from B. subtilis and Zur from $M$. 
tuberculosis unravel that though each Fur subclass responds to different signals, the overall fold of the $\mathrm{N}$-terminal DNA binding domain and the C-terminal dimerization domain is similar [8-10].

Linkage of Fur to the oxidative stress response has been demonstrated in several microorganisms, like Staphylococcus aureus [11] or Mycobacterium tuberculosis [12]. In Synechococcus sp. PCC 7942 and Synechocystis sp. PCC 6803, many iron inducible genes are also influenced by short-term peroxide or methyl viologen stresses [13, 14]. Reactive oxygen species (ROS) are generated during aerobic growth, with the potential to damage almost all cellular components. The formation of these dangerous species is closely related to iron metabolism, since an excess of iron raises the production of ROS through the Fenton reaction [15]. Furthermore, in cyanobacteria iron starvation has shown to be a cause of oxidative stress [16]. Thus, it is not surprising that the cellular responses developed by the organisms to cope with iron starvation and oxidative damage frequently display common elements.

In the filamentous nitrogen-fixing cyanobacterium Anabaena sp. PCC 7120 (also known as Nostoc sp.) three different Fur proteins have been identified: FurA (all1691), FurB (all2473) and FurC (alr0957). In vitro assays suggest that these proteins might be cross-regulated [17]. FurA, as well as FurB, bind to the promoter sequences of the three fur genes, whereas FurC has been proposed to modulate the DNA-binding activity of FurA and FurB.

FurA is a constitutive and relatively abundant protein that seems to be essential in Anabaena sp. [18]. Northern blot analysis shows that under standard growth conditions, mRNAs of furB and furC are less abundant than furA transcripts (our unpublished results). Regulation of FurA takes place at both transcriptional and post-translational levels. Besides a moderate autoregulation, common among fur genes, transcription of Anabaena furA is modulated by the nitrogen regulator NtcA [19] and likely by FurB and FurC [17]. At the post-transcriptional level, an antisense mRNA interferes with the furA transcript [20]. Moreover, post-translational regulation of Fur operated by heme has been reported, since heme binds tightly to FurA impairing its DNA-binding activity [21, 22].

Even though gene regulation is a complex biological process that can happen at several levels, one of the most effective ways for a cell to cope with a change in its environment consists of altering its gene expression. In this work, we show that the expression of the three fur paralogues is strongly affected by oxidative stress. We have used an in vivo approach to demonstrate that, in E. coli, overexpression of FurA and FurB from an inducible promoter prior to oxidative challenge increases cell survival. Moreover, in a purified in vitro system, at saturating concentrations FurB binds to DNA in a sequence-independent fashion and protects DNA from cleavage produced by hydroxyl radicals or DNaseI. Those results unravel a new function for a Fur protein and suggest that in Anabaena, FurA and FurB connect iron regulation to oxidative stress defence.

\section{EXPERIMENTAL PROCEDURES}

\section{Strains and culture conditions.}

The present study was carried out with the filamentous nitrogen-fixing cyanobacterium Anabaena sp. strain PCC 7120 grown in BG-11 medium at $30^{\circ} \mathrm{C}$ [23]. RNAs used in RT-PCR assays were obtained from cultures grown until early-log phase $\left(\mathrm{OD}_{600}=0.4\right)$ and then challenged with $250 \mu \mathrm{M} \mathrm{H}_{2} \mathrm{O}_{2}$ or 1 $\mathrm{mM}$ methyl viologen for $0.5,1,2$ and 4 hours. All the cultures were bubbled vigorously with filtered air.

Overexpression and purification of FurA, FurB and FurC.

Chromosomal DNA from Anabaena sp. was isolated as described previously [24] and used as template for amplification of the furA (all1691), furB (all2473) and furC (alr0957) genes. The PCR products were sequenced and cloned between NcoI and HindIII sites of pET 28a (+). These constructs were used to transform E. coli BL21(DE3) Gold cells to overexpress each Fur protein $[17,25]$.

Recombinant FurA was purified by chromatography through a heparin-sepharose 6 Fast-Flow column (Amersham-Pharmacia) [26]. Recombinant FurB was purified using a heparin-sepharose 6 Fast Flow column (Amersham-Pharmacia) followed by MonoS FPLC column (Pharmacia). Recombinant FurC was purified by heparin-sepharose 6 Fast Flow chromatography followed by MonoQ FPLC column (Pharmacia) as described before [17]. Fur proteins were quantified using the bicinchoninic acid method (BCA ${ }^{\mathrm{TM}}$ Protein Assay Reagent Kit, Pierce). FurA was also quantified spectrophotometrically 
using an extinction coefficient of $13760 \mathrm{M}^{-1} \cdot \mathrm{cm}^{-1}$ at $276 \mathrm{~nm}$ [26]. Quantification was confirmed by densitometric measures in gel using FurA as standard. Sodium dodecyl sulphate polyacrylamide gel electrophoresis (SDS/PAGE) was performed as described by Laemmli [27] using 17\% (w/v) polyacrylamide [30:0.8 $(\mathrm{w} / \mathrm{w})$ acrylamide/bisacrylamide] gels.

\section{Heme-staining assay.}

According to [28], a heme-staining procedure was applied to Fur proteins incubated with heme and separated by SDS/PAGE. Heme solution was prepared dissolving heme (hemin, SIGMA) in $0.1 \mathrm{~N}$ $\mathrm{NaOH}$. The mixture was diluted in $1 \mathrm{mM}$ sodium phosphate $(\mathrm{pH} 7.4)$ to approximately $9.6 \mathrm{M}$.

Aliquots of $5 \mu \mathrm{M}$ FurA, FurB and FurC were incubated with $1 \mathrm{M}$ heme for 5 minutes. Since Anabaena FurA binds heme [21], this reaction was used as positive control of the assay. Lysozyme was used as negative control. Samples were analyzed by SDS/PAGE on a $17 \%(\mathrm{w} / \mathrm{v})$ polyacrilamide gel. The procedure involves staining for heme, distaining and restaining with Coomassie Brilliant Blue for proteins.

Electrophoretic mobility shift assays (EMSA).

DNA fragments of 389-pb containing the upstream sequence of furA were obtained by PCR and further purified using the GFX DNA purification kit (Amersham Biosciences) as described previously [19]. Electrophoretic mobility shift assays were performed as described [25] in a final volume of $20 \mu \mathrm{l}$ using a core binding buffer which contained $10 \mathrm{mM}$ BisTris- $\mathrm{HCl}(\mathrm{pH} 7.5), 40 \mathrm{mM} \mathrm{KCl}, 0.1 \mathrm{mg} / \mathrm{ml}$ BSA, $1 \mathrm{mM}$ DTT, 5\% (v/v) glycerol and $100 \mu \mathrm{M} \mathrm{MnCl}_{2}$. Samples were supplemented with heme at different concentrations. Complexes were separated using $4.8 \%$ polyacrilamide gels. Heme solution was prepared by dissolving heme in a $(1: 9 \mathrm{v} / \mathrm{v}) 0.1 \mathrm{~N} \mathrm{NaOH} / \mathrm{ethanol} \mathrm{solution} \mathrm{and} \mathrm{filtered} \mathrm{through} \mathrm{a}$ $0.2 \mu \mathrm{m}$ filter, as described [21]. Binding assays were carried out in the presence of a 228-bp control DNA fragment from the human apoE gene to demonstrate the specificity of the DNA-binding activity of FurB. Results were processed with a Gel Doc 2000 Image Analyser from Bio-Rad.

RT-PCR analysis.

Anabaena RNA was prepared as described [29], using $40 \mathrm{ml}$ of cells containing $5 \mu \mathrm{g}$ of chlorophyll $a \cdot \mathrm{ml}^{-1}$. Anabaena fur genes were reverse transcribed using the Superscript II Reverse Transcriptase (Invitrogen) and the specific reverse oligonucleotides shown in table 1. The RT-PCR mixture contained $1 \mu \mathrm{g}$ of RNA and $20 \mathrm{pmol}$ of each primer in the annealing buffer $(10 \mathrm{mM}$ Tris- $\mathrm{HCl}(\mathrm{pH}$ $8.0), 1 \mathrm{mM}$ EDTA and $150 \mathrm{mM} \mathrm{KCl}$ ). The total reaction volume was $10 \mu 1$. Samples were incubated $10 \mathrm{~min}$ at $85^{\circ} \mathrm{C}$ to denature RNA followed by $60 \mathrm{~min}$ at $65^{\circ} \mathrm{C}$ to allow annealing of the primer to RNA. After that, 5 pmol of each deoxynucleoside triphosphate (dNTP), $0.2 \mu \mathrm{mol}$ DTT and $200 \mathrm{U}$ Superscript II Reverse Transcriptase (Invitrogen) were added into the RT-PCR buffer (50mM Tris$\mathrm{HCl}(\mathrm{pH} 8.3), 75 \mathrm{mM} \mathrm{KCl}, 3 \mathrm{mM} \mathrm{MgCl}, 20 \mathrm{mM}$ DTT) to a final volume of $20 \mu$ l. Samples were reverse transcribed at $47^{\circ} \mathrm{C}$ for $60 \mathrm{~min}$ followed by $15 \mathrm{~min}$ incubation at $75^{\circ} \mathrm{C}$ to inactivate the reverse transcriptase.

The products were amplified by PCR using $3 \mu \mathrm{l}$ of each cDNA as template. The cycling conditions for the reactions were $3 \mathrm{~min}$ at $95^{\circ} \mathrm{C}$, followed by 25 cycles of $93^{\circ} \mathrm{C}$ for $30 \mathrm{~s}, 50^{\circ} \mathrm{C}$ for $1 \mathrm{~min}$ and $72^{\circ} \mathrm{C}$ for $1 \mathrm{~min}$, followed by extension at $72^{\circ} \mathrm{C}$ for $10 \mathrm{~min}$. Positive control reactions using chromosomic Anabaena sp. DNA as template were run in parallel. PCR products were analyzed in $1 \%$ agarose gel.

In vitro DNA protection assay.

Protection of DNA from damage was performed using pUC19 plasmid DNA (100 ng) in $60 \mu \mathrm{l}$ final volume of $10 \mathrm{mM}$ BisTris- $\mathrm{HCl}(\mathrm{pH} 7.5), 40 \mathrm{mM} \mathrm{KCl}, 0.1 \mathrm{mM} \mathrm{MgCl}_{2}$ and $5 \%$ glycerol (v/v) incubated for 10 minutes with FurB ( 40 pmol or 80 pmol) or BSA ( 40 pmol or 80 pmol) as negative control. When indicated, $100 \mu \mathrm{M} \mathrm{FeCl} 3,400 \mu \mathrm{M}$ EDTA, $150 \mu \mathrm{M} \mathrm{Fe}\left(\mathrm{NH}_{4}\right)_{2}\left(\mathrm{SO}_{4}\right)_{2}$ or $100 \mathrm{mM} \mathrm{H}_{2} \mathrm{O}_{2}$ were added to the reaction mixtures and the reaction allowed to evolve for 20 minutes at room temperature. For protection against DNaseI-mediated cleavage, reactions were assembled as above and $10^{-5}$ units DNaseI- FPLC pure (Amersham Biosciences) added in a buffer containing $0.25 \mathrm{mM}$ $\mathrm{CaCl}_{2}, 0.25 \mathrm{mM} \mathrm{MgCl}$ and $12.5 \%$ glycerol. Samples were incubated for 20 minutes at room temperature. 
Each reaction mixture was divided in two $30 \mu$ l-aliquots, one of them was used as control, while FurB and BSA were removed from the other fraction using a GFX DNA purification kit (Amersham Biosciences). Samples were resolved by electrophoresis in $0.8 \%$ agarose gel and visualized by ethidium bromide staining.

Exposure of E. coli to oxidative stress and disk diffusion assays.

Survival of E. coli cells overexpressing FurA, FurB or FurC was analyzed using methyl viologen (MV) or $\mathrm{H}_{2} \mathrm{O}_{2}$ disk diffusion assays by a modified version of the method described elsewhere [30]. $E$. coli BL21 (DE3) Gold bearing pET-28a (+) was used as negative control. Cells were grown in LB (Luria-Bertani) broth at $37^{\circ} \mathrm{C}$ with shaking. When $\mathrm{OD}_{600}$ of the cultures reached a value of 0.6 , isopropyl- $\beta$-D-thiogalactopyranoside (IPTG) was added at a final concentration of $1 \mathrm{mM}$ and the cultures were grown for another 2 hours. Cells from induced cultures $(200 \mu \mathrm{l})$ were spread onto LB agar containing $1 \mathrm{mM}$ IPTG. Then, aliquots $(2 \mu \mathrm{l})$ of increasing concentrations of $\mathrm{H}_{2} \mathrm{O}_{2}$ (Panreac) or methyl viologen (SIGMA) were spotted on top of Whatman paper disks $(0.5-\mathrm{cm}$ diameter) that were placed over agar plates. Cells were allowed to grow for $24 \mathrm{~h}$ at $37^{\circ} \mathrm{C}$, before measuring the inhibition area around the paper disks.

\section{RESULTS}

Analysis of fur expression by RT-PCR.

Regulation of fur genes under different conditions (iron deficiency, nitrogen-fixing conditions, osmotic stress, saline stress and oxidative stress) has been investigated. When using semiquantitative RT-PCR in those studies, it should be considered the existence of an $\alpha$-furA antisense RNA [31] that can interfere with the results of this assay. To avoid this problem, a specific cDNA for each fur gene has been prepared. Transcription of furA slightly increases under iron starvation as well as in nitrogen-fixing conditions, as reported before [19, 31]. However, these situations do not seem to affect the transcriptional levels of furB and furC. When the cultures were grown under osmotic stress (200 mM sucrose) or salt stress $(100 \mathrm{mM} \mathrm{NaCl})$ the amounts of furA, furB and furC mRNAs remain unchanged (data not shown). Under oxidative stress conditions, transcriptional levels of the three fur genes are affected, being the effect much stronger on furB and furC m-RNAs. In all cases, challenge with $\mathrm{H}_{2} \mathrm{O}_{2}$ induced a faster response than MV (Figure 1).

FurB protects DNA from cleavage produced by hydroxyl radicals or DNaseI .

The dramatic increase of $f u r B$ expression under oxidative challenge, its high isoelectric point (pI 8.7) and the fact that at high concentrations FurB binds to DNA in a sequence-independent fashion (our unpublished results), led us to examine whether purified FurB can afford protection against DNA cleavage. Two situations were tested. On the one hand, DNA was challenged with hydroxyl radicals generated via iron-mediated Fenton reactions, on the other DNA was treated with DNaseI. In those experiments, the combination of Fe (III)/EDTA or Fe (II) and $\mathrm{H}_{2} \mathrm{O}_{2}$ or addition of DNaseI results in the introduction of single-strand breaks that changes supercoiled DNA mobility in agarose gels (figure 2, lanes 3, 6 and 9).

Addition of 40 pmol and 80 pmol of FurB to the reaction mixture containing DNA shows a progressive increase of FurB-DNA complexes, followed by formation of large aggregates that are retained in the wells (figure 2.A.I lanes 4, 5, 7, 8, 10 and 11). FurB binds to supercoiled or linear DNA, suggesting no apparent predilection for either topological form. Removal of FurB before electrophoresis permits to visualize the degree of damage suffered by DNA that was bound to FurB (figure 2.A.II lanes 4, 5, 7, 8, 10 y 11). It can be observed that the presence of increasing amounts of FurB results in a progressive decrease in DNA damage.

In order to check the specificity of the DNA protection by FurB, similar experiments were performed in the presence of FurA (not shown) or BSA (figure2.B) instead of FurB. In those cases DNA is nicked by hydroxyl radicals or DNaseI despite the presence of FurA or BSA. Those results show that FurB binds to and protects DNA from cleavage produced by reactive oxygen species or by DNaseI. 
FurB binds heme.

Free heme is a potential cause of oxidative stress inside the cell [32]. It damages lipids, proteins and DNA through the generation of ROS. In particular, redox-active iron released after degradation of heme plays an important role for heme toxicity [33]. In this way, heme detoxification constitutes a way of ensuring cell survival in the redox environment produced by heme. The ability of Fur and other proteins involved in iron homeostasis to bind heme has been reported previously $[21,22,34]$. In the case of Anabaena FurA, it has been proposed that heme acts as a potential regulator impairing its DNA-binding activity. With the aim to investigate whether FurB and FurC were able to bind heme, a heme-staining procedure was performed as described previously [21]. FurA, used as positive control, FurB, FurC and lysozyme, the latter as negative control, were incubated with heme and the complexes resolved by SDS/PAGE. As shown in figure 3.A, FurB binds heme whereas FurC does not seem to interact with it. This result is in good agreement with the existence of the CP (Cys-Pro) regulatory motif in the sequence of FurA and the CPV (Cys-Pro-Val) one in the sequence of FurB (supplementary material figure S1).

\section{Influence of heme in the DNA-binding activity of FurB.}

Protein-DNA interactions occur between transcription factors and DNA control sequences that lead to gene regulation. They are often the result of extracellular stimuli. The interaction of heme with FurB suggests the possibility that, as in the case of FurA, its DNA-binding activity could be affected. EMSA assays were carried out to assess the influence of heme in the interaction of FurB with the furA promoter. In the absence of heme, FurB binds to $\mathrm{P}_{\text {furA }}$, as previously reported [17]. Addition of heme to the binding assay prior to the incubation with $\mathrm{P}_{\text {furA }}$ inhibits completely the interaction of FurB with DNA, as shown in figure 4.

FurA and FurB increase cell survival under oxidative stress in vivo.

In sight of the results shown to this point, we sought to investigate whether the Fur family is involved in protecting cells from oxidative damage in vivo. If that was the case, the presence of high amounts of Fur in the cells induced by $\mathrm{H}_{2} \mathrm{O}_{2}$ or methyl viologen should increase cell survival. Fur proteins from Anabaena were overexpressed in E. coli from the IPTG-inducible promoter T7 of pET-28a (+) [17]. Those $E$. coli cells were allow to grow on LB-agar plates supplemented with IPTG and Whatman paper disks on top containing increasing concentrations of $\mathrm{H}_{2} \mathrm{O}_{2}$ or MV. Figure 5 shows that when the cells are challenged with $\mathrm{H}_{2} \mathrm{O}_{2}$, overexpression of FurA increases significantly cell survival, as shown by a smaller zone of growth inhibition. Cells overexpressing FurB are also less sensitive to $\mathrm{H}_{2} \mathrm{O}_{2}$ damage. In the MV assays, similar results were observed (figure 6). In both cases, the effect is more evident at high concentrations of $\mathrm{H}_{2} \mathrm{O}_{2}$ or MV. Overexpression of FurC seems not to influence bacterial survival in any condition tested.

\section{DISCUSSION}

The existence of different Fur homologues in distantly related organisms suggests an evolutionary specialization. Although all Fur proteins show common characteristics, there are substantial differences among the signals affecting their affinity for DNA, the sets of target genes or the elements that modulate their transcription. To get a better understanding of the roles of fur paralogues in Anabaena, we have investigated the regulation of those genes under different situations, as well as the behaviour of their protein products in face of agents that could induce DNA damage.

While osmotic and saline stresses did not affect the transcription of fur genes, oxidative challenge lead to increased levels of the three fur transcripts, particularly of furB and furC. This indicates that the raise of the transcription is not a general response to any stress and are consistent with previous reports linking iron-responsive gene expression and oxidative stress response [13, 14, 16, 35]. In many microorganisms, Fur proteins regulate the expression of genes involved in the concerted defence against oxidative stress, such as superoxide dismutase or catalase. In the unicellular cyanobacterium Synechocystis sp. PCC 6803, the Fur-like Slr1738 plays a regulatory role in the induction of the potent antioxidant gene $\operatorname{sll} 1621(\mathrm{ahpC})[14,35]$. Both genes are strongly induced by methyl viologen or peroxide treatment and are located contiguously in the genome. 
In addition to ROS generated by the Fenton's reaction and by the adventitious delivery of electrons from the photosynthetic and respiratory pathways to oxygen, the potential of heme to catalyse free radical formation has to be considered. Histidine residues and sometimes cysteine-proline (CP) [36] or cysteine-proline-valine (CPV) [37] sequences, have been proposed to be involved in the interaction between heme and proteins. A common feature of the Fur family is the presence of a histidine-rich motif that is involved in metal binding. Moreover, FurA and FurB from Anabaena contain in their sequences CP or CPV motifs, respectively, located in their carboxyl ends (figure S1), which accounts for the ability to bind metal and dimerise $[8,38]$. The CP or CPV sequences are thought to be regulatory elements for non-covalent binding of heme to proteins [37]. Several functions are proposed for these sequences. They could act, for instance, as a secondary heme binding sites or contribute to the complex formation by increasing the concentration of heme around them [21]. The presence of the $\mathrm{CP}$ motif in the sequence of FurA and CPV one in the sequence of FurB is in good agreement with the ability of these proteins to bind heme, while FurC, that lacks any of these signatures, does not interact with it.

In Rhizobiales, heme regulates the degradation of the Fur-related response regulator Irr, whose stability depends on the iron status [34]. In the case of FurA and FurB, degradation products are not detected after incubation with heme, suggesting that heme plays a different role in Irr than in Fur proteins.

Different mechanisms of redox control of gene expression have evolved to adapt rapidly to redox changes within bacteria. The regulator FixL utilizes heme as co-repressor to elicit a conformational change in response to oxygen [39]. Similarly, the interaction of heme with FurA and FurB could produce a conformational change providing a mechanism of post-translational regulation. This model is in good agreement with the fact that heme impairs the DNA binding activity of FurB and, as previously reported, of FurA [21]. Fur regulation seems to be a complex process which involves not only the existence of $\mathrm{Fe}(\mathrm{II})$ and reducing conditions [18]. The DNA-binding ability of Fur, and consequently, its functionality, is impaired also by the dimerisation rate and by heme binding.

The contexts of the fur homologues in Anabaena are rather different that those of the corresponding ortologues in Synechocystis sp. PCC 6803 . While the peroxide-dependent slr1738 is contiguos to $a h p C$, the orthologue to $f u r B$, sll1937, lies divergently next to $z n t C$, that codes for a component of an $\mathrm{ABC}$ type zinc uptake transporter [40]. Analysis of the sequences flanking furB and furC in Anabaena (Cyanobase: http://bacteria.kazusa.or.jp/cyano/) shows that the ORFs contiguous to those fur genes encode hypothetical or unknown proteins. On the other hand, the closest homologues to $\operatorname{ahpC}$ and $z n t C$, the all1541 and all0833 genes, are located next to other ORFs unrelated to the fur family.

Our results demonstrate that FurB protects DNA from cleavage produced by hydroxyl radicals or DNaseI. In addition, overexpression of FurA and FurB in E. coli increases survival when the cells are stressed with hydrogen peroxide or methyl viologen. Although overexpression of FurC does not seem to influence cell survival, it could be hypothesized that FurC might play a role in the regulation of cyanobacterial metabolic processes that are absent in E. coli, such as those related to photosynthesis. In fact, expression of fur $C$ is enhanced specifically by addition of methyl viologen, a superoxide anion-generating reagent. In photosynthetic organisms, the most important production of superoxide anion takes place in the photosynthetic electron transport chain, when molecular oxygen adventitiously oxidizes PSI [41]. Thus, FurC might be involved in the protection of the photosynthetic proteins from oxidative damage.

In the case of FurA, it might increase cell survival modulating a set of genes involved in the detoxification of ROS. Indeed, FurA recognises the regulatory regions of several key genes related to redox homeostasis, such as gor and a number of thioredoxins [42] suggesting that FurA could coordinate the cellular response to oxidative stress.

Overexpression of FurB possibly increases cell survival under oxidative challenge avoiding DNA damage by unspecific binding. The cationic isoelectric point of FurB (theoretical $\mathrm{pI}=8.7$ ), together with its high affinity to DNA, its ability to bind heme and its DNA-protective activity suggest that FurB could act as a Dps-like protein [43]. In addition to Dps, there are other bacterial, relatively nonspecific DNA-binding proteins that exert different functions depending on the level of expression. $\mathrm{H}-$ NS is one of the most abundant chromatin-associated proteins [44]. At sub-saturating concentrations, H-NS exhibits a preferred DNA-binding site modulating transcription of the proU operon. However, when present in saturating concentrations, H-NS binds to DNA in a sequence-independent fashion. 
This could be the case of furB, whose transcription is triggered by reactive oxygen species. Under those conditions, the level of FurB would be high enough to coat DNA unspecifically and prevent oxidative damage. To our knowledge, this is the first evidence that a Fur protein protects DNA from cleavage by direct binding.

\section{REFERENCES}

1 Andrews, S. C., Robinson, A. K. and Rodríguez-Quiñones, F. (2003) Bacterial iron homeostasis. FEMS Microbiol Rev 27, 215-237

2 Bagg, A. and Neilands, J. B. (1987) Ferric uptake regulation protein acts as a repressor, employing iron (II) as a cofactor to bind the operator of an iron transport operon in Escherichia coli. Biochemistry 26, 5471-5477

3 Patzer, S. I. and Hantke, K. (1998) The ZnuABC high-affinity zinc uptake system and its regulator Zur in Escherichia coli. Mol Microbiol 28, 1199-1210

4 Díaz-Mireles, E., Wexler, M., Sawers, G., Bellini, D., Todd, J. D. and Johnston, A. W. (2004) The Fur-like protein Mur of Rhizobium legominosarum is a $\mathrm{Mn}(2+)$-responsive transcriptional regulator. Microbiology 150, 1447-1456

5 Ahn, B. E., Cha, J., Lee, E. J., Han, A. R., Thompson, C. J. and Roe, J. H. (2006) Nur, a nickel-responsive regulator of the Fur family, regulates superoxide dismutases and nickel transport in Streptomyces coelicolor. Mol Microbiol 59, 1848-1858

6 Bsat, N., Herbig, A., Casillas-Martinez, L., Setlow, P. and Helmann, J. D. (1998) Bacillus subtilis contains multiple Fur homologues: identification of the iron uptake (Fur) and peroxide regulon (PerR) repressors. Mol Microbiol 29, 189-198

7 Hamza, I., Chauhan, V. S., Hassett, R. and O' Brian, M. R. (1998) The bacterial Irr protein is required for coordination of heme biosynthesis with iron availability. J Biol Chem 273, 21669-21674

8 Pohl, E., Haller, J. C., Mijovilovich, A., Meyer-Klaucke, W., Garman, E. and Vasil, M. L. (2003) Architecture of a protein central to iron homeostasis: crystal structure and spectroscopic analysis of the ferric uptake regulator. Mol Microbiol 47, 903-915

9 Lucarelli, D., Russo, S., Garman, E., Milano, A., Meyer-Klaucke, W. and Pohl, E. (2007) Crystal structure and function of the zinc uptake regulator FurB from Mycobacterium tuberculosis. J Biol Chem 282, 9914-9922

10 Traore, D. A., El Ghazouani, A., Ilango, S., Dupuy, J., Jacquamet, L., Ferrer, J. L., CauxThang, C., Duarte, V. and Latour, J. M. (2006) Crystal structure of the apo-PerR-Zn protein from Bacillus subtilis. Mol Microbiol 61, 1211-1219

11 Horsburgh, M. J., Ingham, E. and Foster, S. J. (2001) In Staphylococcus aureus, Fur is an interactive regulator with PerR, contributes to virulence, and is necessary for oxidative stress resistance through positive regulation of catalase and iron homeostasis. J Bacteriol 183, 468475

12 Pagan-Ramos, E., Song, J., McFalone, M., Mudd, M. H. and Deretic, V. (1998) Oxidative stress response and characterization of the $\operatorname{oxyR}$ - ahpC and furA-katG loci in Mycobacterium marinum. J Bacteriol 180, 4856-4864

13 Yousef, N., Pistorius, E. K. and Michel, K. P. (2003) Comparative analysis of idiA and isiA transcription under iron starvation and oxidative stress in Synechococcus elongatus PCC 7942 wild-type and selected mutants. Arch Microbiol 180, 471-483

14 Li, H., Singh, A. K., McIntyre, L. M. and Sherman, L. A. (2004) Differential gene expression in response to hydrogen peroxide and the putative PerR regulon of Synechocystis sp. strain PCC 6803. J Bacteriol 186, 3331-3345

15 Imlay, J. A. (2003) Pathways of oxidative damage. Annu Rev Microbiol 57, 395-418

16 Latifi, A., Jeanjean, R., Lemeille, S., Havaux, M. and Zhang, C. C. (2005) Iron starvation leads to oxidative stress in Anabaena sp. strain PCC 7120. J Bacteriol 187, 6596-6598

17 Hernández, J. A., López-Gomollón, S., Bes, M. T., Fillat, M. F. and Peleato, M. L. (2004) Three fur homologues from Anabaena sp. PCC 7120: exploring reciprocal protein-promoter recognition. FEMS Microbiol Lett 236, 275-282 
Hernández, J. A., López-Gomollón, S., Muro-Pastor, A., Valladares, A., Bes, M. T., Peleato, M. L. and Fillat, M. F. (2006) Interaction of FurA from Anabaena sp. PCC 7120 with DNA: A reducing environment and the presence of $\mathrm{Mn} 2+$ are positive effectors in the binding to $i s i B$ and furA promoters. Biometals 19, 259-268

19 López-Gomollón, S., Hernández, J. A., Wolk, C. P., Peleato, M. L. and Fillat, M. F. (2007) Expression of furA is modulated by NtcA and strongly enhanced in heterocysts of Anabaena sp. PCC 7120. Microbiology 153, 42-50

20 Hernández, J. A., Muro-Pastor, A. M., Flores, E., Bes, M. T., Peleato, M. L. and Fillat, M. F. (2006) Identification of a furA cis antisense RNA in the cyanobacterium Anabaena sp. PCC 7120. J Mol Biol 355, 325-334

21 Hernández, J. A., Peleato, M. L., Fillat, M. F. and Bes, M. T. (2004) Heme binds to and inhibits the DNA-binding activity of the global regulator FurA from Anabaena sp. PCC 7120. FEBS Lett 577, 35-41

22 Smith, A., Hooper, N. I., Shipulina, N. and Morgan, W. T. (1996) Heme binding by a bacterial repressor protein, the gene product of the ferric uptake regulation (fur) gene of Escherichia coli. J Protein Chem 15, 575-583

23 Rippka, R. (1988) Isolation and purification of cyanobacteria. Methods Enzymol 167, 3-27

24 Cai, Y. P. and Wolk, C. P. (1990) Use of a conditionally lethal gene in Anabaena sp. strain PCC 7120 to select for double recombinants and to entrap insertion sequences. J Bacteriol 172, 3138-3145

25 Bes, M. T., Hernández, J. A., Peleato, M. L. and Fillat, M. F. (2001) Cloning, overexpression and interaction of recombinant Fur from the cyanobacterium Anabaena PCC 7119 with isiB and its own promoter. FEMS Microbiol Lett 194, 187-192

26 Hernández, J. A., Bes, M. T., Fillat, M. F., Neira, J. L. and Peleato, M. L. (2002) Biochemical analysis of the recombinant Fur (ferric uptake regulator) protein from Anabaena PCC 7119: factors affecting its oligomerization state. Biochem J 366, 315-322

27 Laemmli, U. K. (1970) Cleavage of structural proteins during the assembly of the head of bacteriophage T4. Nature (London) 227, 680-685

28 Granath, W. O. J. (1988) Identification of hemeproteins in the hemolymph of Biomphalaria glabrata (Gastropoda) separated by sodium dodecyl sulfate-polyacrylamide gel electrophoresis. J Invertebr Pathol 52, 43-48

29 Muro-Pastor, A. M., Valladares, A., Flores, E. and Herrero, A. (2002) Mutual dependence of the expression of the cell differentiation regulatory protein HetR and the global nitrogen regulator NtcA during heterocyst development. Mol Microbiol 44, 1377-1385

30 Christman, M. F., Morgan, R. W., Jacobson, F. S. and Ames, B. N. (1985) Positive control of a regulon for defenses against oxidative stress and some heat-shock proteins in Salmonella typhimurium. Cell 41, 753-762

31 Hernandez, J. A., Muro-Pastor, A. M., Flores, E., Bes, M. T., Peleato, M. L. and Fillat, M. F. (2006) Identification of a furA cis antisense RNA in the cyanobacterium Anabaena sp. PCC 7120. J Mol Biol 355, 325-334

32 Gutteridge, J. M. and Smith, A. (1988) Antioxidant protection by haemopexin of haemstimulated lipid peroxidation. Biochem J 256, 861-865

33 Kumar, S. and Bandyopadhyay, U. (2005) Free heme toxicity and its detoxification systems in humans. Toxicol Lett 157, 175-188

34 Qi, Z., Hamza, I. and O' Brian, M. R. (1999) Heme is an effector molecule for iron-dependent degradation of the bacterial iron-response regulator (Irr) protein. Proc Natl Acad Sci U S A 96, 13056-13061

35 Kobayashi, M., Ishizuka, T., Katayama, M., Kanehisa, M., Bhattacharyya-Pakrasi, M., Pakrasi, H. B. and Ikeuchi, M. (2004) Response to oxidative stress involves a novel peroxiredoxin gene in the unicellular cyanobacterium Synechocystis sp. PCC 6803. Plant Cell Physiol 45, 290-299

36 Ogawa, K., Sun, J., Taketani, S., Nakajima, O., Nishitani, C., Sassa, S., Hayashi, N., Yamamoto, M., Shibahara, S., Fujita, H. and Igarashi, K. (2001) Heme mediates derepression of Maf recognition element through direct binding to transcription repressor Bach1. Embo J 20, 2835-2843 
37 Zhang, L. and Guarente, L. (1995) Heme binds to a short sequence that serves a regulatory function in diverse proteins. Embo J 14, 313-320

38 Coy, M. and Neilands, J. B. (1991) Structural dynamics and functional domains of the fur protein. Biochemistry 30, 8201-8210

39 Bauer, C. E., Elsen, S. and Bird, T. H. (1999) Mechanisms for redox control of gene expression. Annu Rev Microbiol 53, 495-523

40 Pakrasi, H. B., Ogawa, T. and Bhattacharrya-Pakrasi, M. (2001) Transport of Metals: a key process in oxygenic photosynthesis. In Regulation of Photosynthesis (Aro, E. M. and Anderson, B. E., eds.), pp. 253-264, Kluwer Academic Publishers

41 Asada, K. (1994) Production and action of active oxygen species in photosynthesis tissues. In Causes of photooxidative stress and amelioration of defense systems in plants (Foyer, C. H. and Mullineaux, P. M., eds.), pp. 77-104, CRC Press, Boca Raton, Fla.

42 López-Gomollón, S., Hernández, J. A., Pellicer, S., Espinosa, V., Peleato, M. L. and Fillat, M. F. (2007) Cross-talk between iron and nitrogen regulatory networks in Anabaena (Nostoc) sp. PCC 7120: identification of overlapping genes in FurA and NtcA regulons. J Mol Biol 374, 267-281

43 Peña, M. M. and Bullerjahn, G. S. (1995) The DpsA protein of Synechococcus sp. strain PCC 7942 is a DNA-binding hemoprotein. Linkage of the Dps and bacterioferritin protein families. J Biol Chem 270, 22478-22482

44 Tupper, A. E., Owen-Hughes, T. A., Ussery, D. W., Santos, D. S., Ferguson, D. J., Sidebotham, J. M., Hinton, J. C. and Higgins, C. F. (1994) The chromatin-associated protein H-NS alters DNA topology in vitro. Embo J 13, 258-268

\section{ACKNOWLEDGEMENTS}

The authors thank Dr Silvia Pellicer for helpful discussions. This work was supported by grant BFU2006-03454/BMC (Programa nacional de I+D, Ministerio de Educación y Ciencia, Spain). 


\section{FIGURE LEGENDS}

Figure 1. RT-PCR analysis of the fur family transcription under oxidative stress conditions. Expression of furA, furB and furC under oxidative stress conditions produced by $250 \mu \mathrm{M} \mathrm{H}_{2} \mathrm{O}_{2}$ and 1 $\mathrm{mM}$ methyl viologen. Transcripts were analyzed at different times by RT-PCR. PCR reactions with the corresponding oligonucleotides using chromosomic Anabaena sp. DNA as template were carried out as positive controls.

Figure 2. In vitro DNA protection assay.

The introduction of a single-stranded break in the plasmid DNA converts it to a nicked form changing its mobility in an agarose gel. As the substrate was used $100 \mathrm{ng}$ of pUC plasmid. When indicated, Fe (III)/EDTA or Fe (II) and $\mathrm{H}_{2} \mathrm{O}_{2}$ or DNaseI were added to the samples as denoted below the panels. FurB (40 pmol and $80 \mathrm{pmol})$ as is shown in panel A, and BSA (40 pmol and 80 pmol) as negative control, in panel B, were added to the samples. After incubation for 20 minutes at room temperature, the reactions were stopped and proteins were removed from half of each sample. DNA was visualized by electrophoresis in $0.8 \%$ agarose gel. $\mathrm{N}$ : nicked DNA, L: linear DNA, SC: supercoiled DNA.

Figure 3. Heme binding and staining assay.

(A) $5 \mu \mathrm{M}$ of each protein were incubated 5 minutes with heme. FurA (MW= $17.2 \mathrm{KDa}$ ) was used as positive control or the experiment. Lysozyme $(\mathrm{MW}=14.3 \mathrm{KDa})$ was used as a control of non-specific binding of heme to protein. Samples were analyzed in SDS/PAGE and a specific heme-staining assay was performed [28]. (B) After that, proteins were detected with Coomassie blue staining. The sizes of the molecular mass markers are indicated on the left side. Note that the samples are loaded using a non-reducing loading buffer, thus several oligomeric forms of FurA, FurB and FurC could be detected.

\section{Figure 4. Inhibition of in vitro DNA binding activity of FurB by heme.}

Electrophoretic mobility shift assay of FurB to a 389-bp DNA fragment containing the Anabaena sp. furA promoter. A fragment of exon IV from the human apoE gene was used as non-specific competitor DNA. Lane 1: free DNA. Lane 2: $500 \mathrm{nM}$ FurB. Lane 3: Reaction mixture in the presence of 1:5 FurB /heme concentration ratio ( $500 \mathrm{nM}$ FurB and $2.5 \mu \mathrm{M}$ heme). Lane 4: Reaction mixture in the presence of 1:8 FurB /heme concentration ratio (500 nM FurB and $4 \mu \mathrm{M}$ heme).

Figure 5. $\mathrm{H}_{2} \mathrm{O}_{2}$ disk assay.

In the assay, LB-agar plates were inoculated from liquid cultures of $E$. coli strains overexpressing FurA, FurB and FurC in the presence of $1 \mathrm{mM}$ IPTG. E. coli containing pET-28a(+) was used as negative control. Sensitivity to $\mathrm{H}_{2} \mathrm{O}_{2}$ was measured by the zone of growth inhibition (diameter). (A) Effect of $\mathrm{H}_{2} \mathrm{O}_{2}$ on growth of $E$. coli bearing (a) pET-28a(+) plasmid (negative control), (b) fur A cloned into pET-28a(+), (c) furB cloned into pET-28a(+), (d) furC cloned into pET-28a(+). $\mathrm{H}_{2} \mathrm{O}_{2}$ concentration assayed: (1) $9.7 \mathrm{M}$, (2) $4.85 \mathrm{M}$, (3) $1.94 \mathrm{M}$, (4) $0.97 \mathrm{M}$ and (5) $0.48 \mathrm{M}$. (B) The zone of inhibition at each concentration of $\mathrm{H}_{2} \mathrm{O}_{2}$ of negative control (black bars) and furA (light grey bars), furB (white bars), furC (dark grey bars) cloned into pET-28a $(+)$ was measured five times, and the mean values were represented. The error bars are the standard errors of the mean value.

Figure 6. Methyl viologen disk assay.

In the assay, LB-agar plates were inoculated from liquid cultures of E. coli strains overexpressing FurA, FurB and FurC in the presence of $1 \mathrm{mM}$ IPTG. Sensitivity to MV was measured by the zone of growth inhibition (diameter). (A) Effect of methyl viologen on growth of E. coli bearing (a) pET$28 \mathrm{a}(+)$ plasmid (negative control), (b) furA cloned into pET-28a(+), (c) furB cloned into pET-28a(+), (d) furC cloned into pET-28a(+). MV concentration assayed: (1) $1000 \mathrm{mM}$, (2) $500 \mathrm{mM}$, (3) $100 \mathrm{mM}$, (4) $75 \mathrm{mM}$, (5) $50 \mathrm{mM}$, (6) $25 \mathrm{mM}$ and (7) $10 \mathrm{mM}$. (B) The zone of inhibition at each concentration of MV of negative control (black bars) and furA (light grey bars), furB (white bars), furC (dark grey bars) cloned into pET-28a $(+)$ was measured five times, and the mean values were represented. The error bars are the standard errors of the mean value. 
Biochemical Journal Immediate Publication. Published on 22 Oct 2008 as manuscript BJ20081066

Table 1. Oligonucleotides used in this work

\begin{tabular}{ccc}
\hline Primer & Sequence $\left(5^{\prime}-\mathbf{3}^{\prime}\right)$ & Description \\
\hline FurN & ATTACCATGGCTGTCTACAC & RT-PCR of \\
FurC & CTTGTAAGCTTACCAGGTGC & furA \\
\hline all2473-N & ATAGTCCATGGGAGCCATAC & RT-PCR of \\
all2473-C & CAGTAGTTTAAGCTTTTGACTA & furB \\
\hline alr0957-N & GACATAACCATGGAGCAACAG & RT-PCR of \\
alr0957-C & CTACTCCCAAGCTTTCTACTC & furC \\
\hline
\end{tabular}


Biochemical Journal Immediate Publication. Published on 22 Oct 2008 as manuscript BJ20081066

Figure 1. RT-PCR analysis of fur family expression under oxidative stress conditions

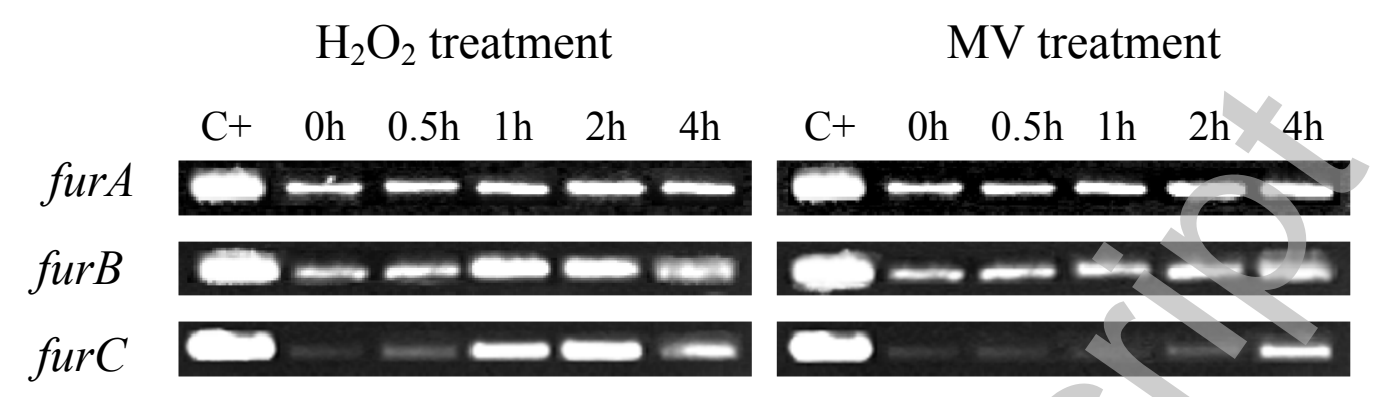


Figure 2. In vitro DNA protection assay

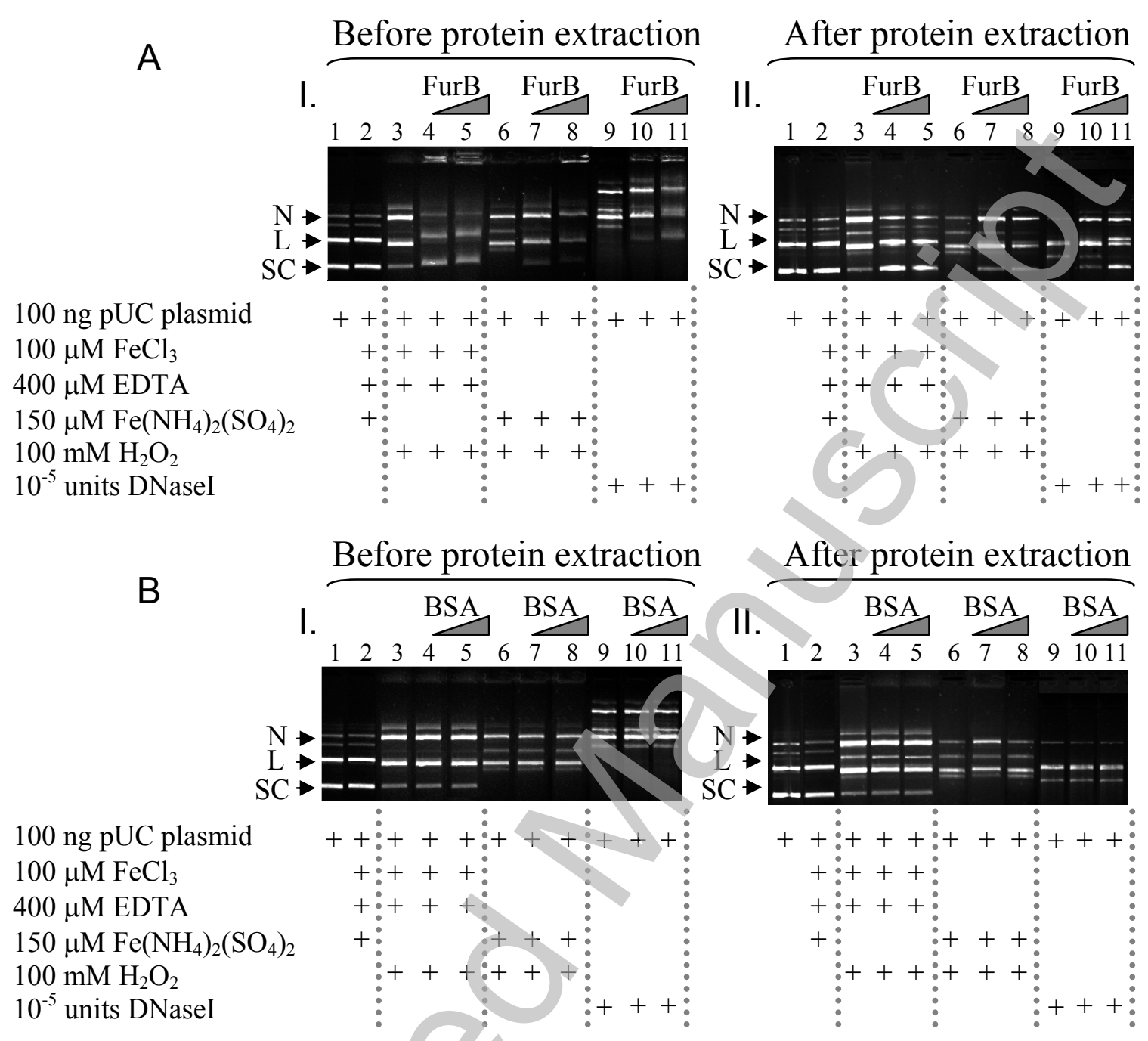


Figure 3. Heme binding and staining assay

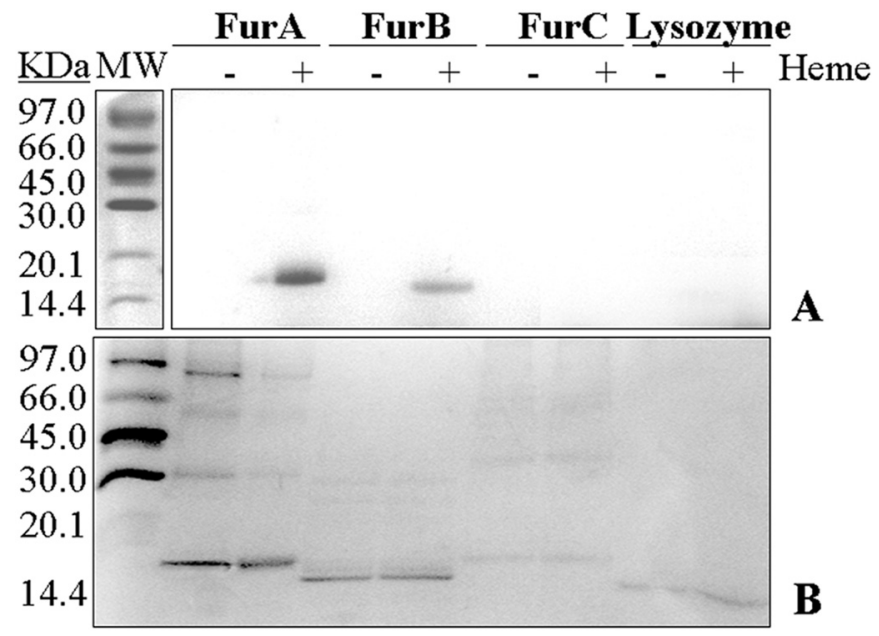


Figure 4. Inhibition of in vitro DNA binding activity of FurB by heme

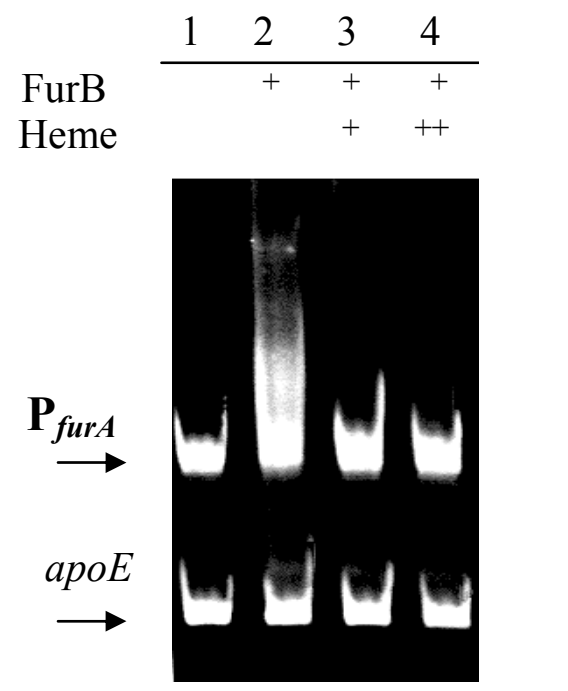


Figure 5. $\mathrm{H}_{2} \mathrm{O}_{2}$ disk assay

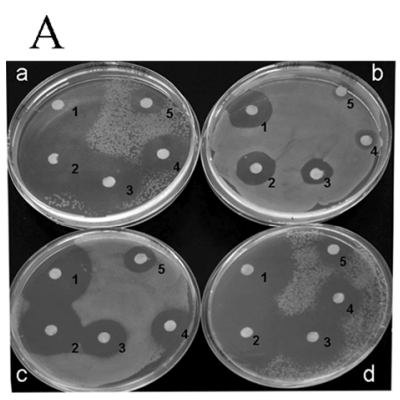

$\mathrm{B}$

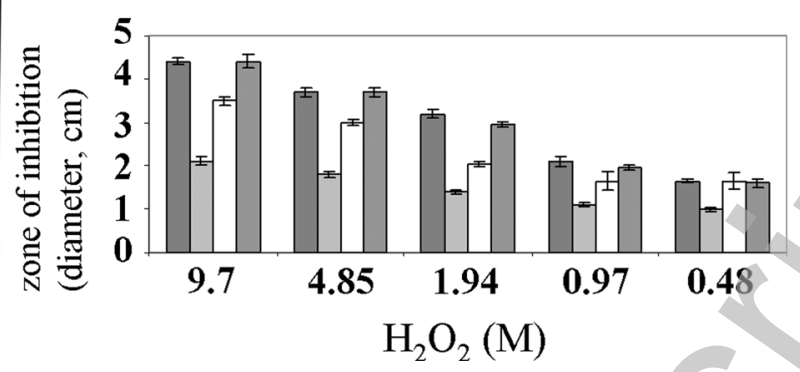


Figure 6. Methyl viologen disk assay

A

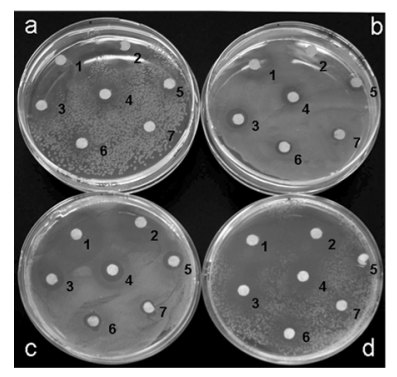

B

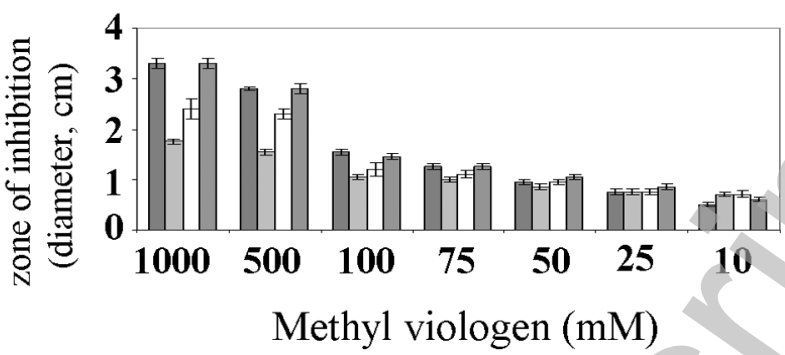

